\title{
Solution of Material Selection Problem Using Fuzzy Axiomatic Design and DEMATEL Methods
}

\author{
G. CANDAN*, S. KIR AND H.R. YAZGAN \\ Sakarya University, Department of Industrial Engineering, 54050 Sakarya, Turkey
}

\begin{abstract}
Material selection is an important task for dental healthcare. A teeth can be filled with several filling materials such as a silver amalgam (mixed with silver, tin, zinc, and copper), a plastic, a porcelain, and composites. Choosing the best filling material is a complex process for a dentist because of human healthcare. There are many criteria for the selection process such as aesthetics, resistant, strength, rigid, long life, low cost, healthy. One of the novelty of this research that is the first research in the field of selecting dental material and another novelty that is used fuzzy axiomatic design and fuzzy DEMATEL methods together first time. First of all, the most effective criteria are determined in order to choose the best material. The weights of the criteria are determined using interaction among themselves. Interactions among criteria are found using DEMATEL method with taking into consideration of dentists' experiences on a teeth filling. Secondly, evaluating alternative materials is carried out using fuzzy axiomatic design method. The results illustrate that the proposed approach is suitable for selecting materials of a teeth filling. We believe that the approach can be applicable for other material selection problems.
\end{abstract}

DOI: 10.12693 /APhysPolA.131.24

PACS/topics: $89.20 . \mathrm{Kk}, 89.65 . \mathrm{Gh}$

\section{Introduction}

Dental filling is a restoration process for teeth damages due to a tooth decay. Dental health is directly related to healthcare, so selecting the best filling material is an important task. There are a few filling materials such as $\mathrm{Ag}-\mathrm{Sn}$ (amalgam), $\mathrm{Ni}-\mathrm{Cr}$ composites, $\mathrm{Co}-\mathrm{Cr}$ composites, $\mathrm{Au}-\mathrm{Pt}$ composites, composite resin (plastic- $\mathrm{SiO}_{2}$ ). Primarily effective criteria should be determined in order to choose a suitable material. Long life (C1), healthy $(\mathrm{C} 2)$, repairability $(\mathrm{C} 3)$, strength $(\mathrm{C} 4)$, heat resistance (C5), aesthetic (C6), formability (C7), corrosion resistance (C8), biocompatibility (C9), cost-effective (C10), easy access (C11), easy storage (C12), eco-friendly (C13), versatility (C14), rigid (C15) were considered for general filling material selecting problems. A novel approach is proposed to select a dental filling material in this study. We believe that our proposed model consists of two novelties in the literature. Our paper is first study deal with a dental filling material selection problem. The other one is that we used fuzzy axiomatic design and fuzzy DEMATEL methods together first time.

According to the literature review, 15 material selection criteria were determined to use for dental filling. Unlike other studies, the weights of these criteria were determined by considering interaction among themselves. When determining the weights of criteria, interactions of criteria were evaluated separately. The process of evaluating the criteria interactions was performed with specialist dentists in order to obtain useable criteria weights. The fuzzy decision making trial and evaluation

*corresponding author; e-mail: gcandan@sakarya.edu.tr laboratory (DEMATEL) method was preferred to determine criteria weights, because of considering criteria interactions and the criteria were fuzzy. After determining the weights of selection criteria, making a selection from the alternative materials is required. The evaluation process of the alternative materials should be made as fuzzy and based on the experience by the reason of fuzzy criteria. Based on this, fuzzy axiomatic design method was preferred to making a selection from the alternative materials. Triangular fuzzy expression design patterns were prepared with the same specialist dentists.

\section{Method and application}

\subsection{Determining the criteria weights by fuzzy DEMATEL method}

DEMATEL method is presented by Fontela and Gabus [1] which involves the complex causal relationships between criteria to create a structural model and a comprehensive method for analyzing. DEMATEL method divides the criteria into two groups particularly as a cause and an effect groups. Determining affecting or being affected criteria is an important step to solve complex problems. In our study, we calculated the importance of our decision criteria weights using the finite-difference (FD) method. The steps of the determining weights of criteria were the following:

Step 1: The triangular fuzzy numbers were determined corresponding to fuzzy expressions as in Table I.

Step 2: 88 different linguistic direct relation matrices were constituted by four dentists by considering the determined 15 criteria in our problem. One of them is shown in Table II. Then these matrices were converted to triangular fuzzy numeric form as Eq. (1) where $\tilde{z}_{i j}=\left(z_{i j, l} z_{i j, m} z_{i j, u}\right)$. 
TABLE I

Triangular fuzzy numbers corresponding to fuzzy expressions.

\begin{tabular}{c|c}
\hline \hline Fuzzy expressions & $\begin{array}{c}\text { Triangular fuzzy numbers } \\
(l, m, u)\end{array}$ \\
\hline Very high influence (VH) & $(0.75,1.0,1.0)$ \\
High influence (H) & $(0.5 .0 .75,1.0)$ \\
Low influence (L) & $(0.25,0.5 .0 .75)$ \\
Very low influence (VL) & $(0,0.25,0.50)$ \\
No influence (NO) & $(0,0,0.25)$
\end{tabular}

TABLE II

An example of linguistic direct relation matrix for $\mathrm{Ag}-\mathrm{Sn}$.

\begin{tabular}{|c|c|c|c|c|c|c|c|c|c|c|c|c|c|c|c|}
\hline $\mathrm{RM}_{1} \mathrm{E}_{1}$ & C1 & $\mathrm{C} 2$ & C3 & $\mathrm{C} 4$ & C5 & C6 & C7 & C8 & C9 & C10 & C11 & C12 & C13 & C14 & $\mathrm{C} 15$ \\
\hline C1 & - & $\mathrm{VH}$ & VL & $\mathrm{H}$ & VL & VL & VL & $\mathrm{H}$ & L & $\mathrm{VH}$ & NO & NO & $\mathrm{NO}$ & VL & $\mathrm{NC}$ \\
\hline $\mathrm{C} 2$ & VL & & $\mathrm{VH}$ & $\mathrm{VH}$ & $\mathrm{H}$ & $\mathrm{VH}$ & $\mathrm{H}$ & VL & $\mathrm{H}$ & $\mathrm{H}$ & NO & VL & NO & $\mathrm{L}$ & NO \\
\hline $\mathrm{C} 3$ & $\mathrm{H}$ & $\mathrm{VH}$ & - & $\mathrm{H}$ & $\mathrm{H}$ & $\mathrm{H}$ & $\mathrm{VH}$ & L & L & $\mathrm{VL}$ & NO & $\mathrm{H}$ & NO & $\mathrm{L}$ & $\mathrm{NO}$ \\
\hline $\mathrm{C} 4$ & $\mathrm{VL}$ & $\mathrm{H}$ & VL & - & $\mathrm{L}$ & VL & NO & NO & VL & VL & NO & $\mathrm{H}$ & $\mathrm{NO}$ & $\mathrm{NO}$ & NO \\
\hline C5 & $\mathrm{VL}$ & $\mathrm{H}$ & VL & $\mathrm{VH}$ & - & $\mathrm{VH}$ & L & NO & $\mathrm{VH}$ & $\mathrm{H}$ & NO & L & $\mathrm{NO}$ & $\mathrm{L}$ & NO \\
\hline C6 & $\mathrm{H}$ & $\mathrm{VH}$ & $\mathrm{L}$ & $\mathrm{H}$ & VL & - & $\mathrm{L}$ & $\mathrm{NO}$ & VL & $\mathrm{VH}$ & $\mathrm{NO}$ & VL & NO & VL & NO \\
\hline C7 & L & $\mathrm{VH}$ & $\mathrm{H}$ & $\mathrm{H}$ & VL & L & - & NO & L & $\mathrm{H}$ & $\mathrm{NO}$ & VH & NO & VL & NO \\
\hline C8 & $\mathrm{VL}$ & VL & $\mathrm{H}$ & $\mathrm{L}$ & VL & $\mathrm{H}$ & $\mathrm{H}$ & - & VL & VL & $\mathrm{NO}$ & VL & |NO & VL & NO \\
\hline C9 & $\mathrm{VL}$ & $\mathrm{H}$ & $\mathrm{VL}$ & VL & $\mathrm{H}$ & $\mathrm{H}$ & L & NO & - & $\mathrm{VH}$ & $\mathrm{NO}$ & $\mathrm{H}$ & NO & $\mathrm{NO}$ & NO \\
\hline C10 & $\mathrm{VL}$ & VL & $\mathrm{VL}$ & $\mathrm{L}$ & $\mathrm{L}$ & VL & VL & VL & $\mathrm{VH}$ & - & $\mathrm{NO}$ & VH & $\mathrm{NO}$ & $\mathrm{NO}$ & NO \\
\hline C11 & L & $\mathrm{H}$ & $\mathrm{H}$ & $\mathrm{VH}$ & VH & $\mathrm{H}$ & VH & VL & VH & $\mathrm{L}$ & - & $\mathrm{L}$ & $\mathrm{VH}$ & VL & NO \\
\hline $\mathrm{C} 12$ & VL & $\mathrm{H}$ & L & $\mathrm{L}$ & $\mathrm{L}$ & VL & $\mathrm{L}$ & NO & L & $\mathrm{H}$ & $\mathrm{NO}$ & $\begin{array}{lll}- & \\
\end{array}$ & $\mathrm{NO}$ & VL & NO \\
\hline $\mathrm{C} 1$ & $\mathrm{VH}$ & $\mathrm{H}$ & $\mathrm{VL}$ & $\mathrm{VH}$ & VL & $\mathrm{L}$ & VL & VL & $\mathrm{L}$ & $\mathrm{VH}$ & VH & L & - & VL & $\mathrm{VH}$ \\
\hline C14 & $\mathrm{H}$ & $\mathrm{H}$ & VL & $\mathrm{L}$ & $\mathrm{H}$ & VL & VL & VL & L & $\mathrm{L}$ & $\mathrm{NO}$ & $\mathrm{L}$ & $\mathrm{NO}$ & - & $\mathrm{NO}$ \\
\hline C15 & $V F$ & 11 & $\sqrt{1}$ & & & & & & & & $\mathrm{NO}$ & & NO & VL & \\
\hline
\end{tabular}

$$
\tilde{Z}=\left[\begin{array}{cccc}
0 & \tilde{z}_{12} & \ldots & \tilde{z}_{1 n} \\
\tilde{z}_{21} & 0 & \ldots & \tilde{z}_{2 n} \\
\vdots & \vdots & \ddots & \vdots \\
\tilde{z}_{n 1} & \tilde{z}_{n 2} & \ldots & 0
\end{array}\right]
$$

Step 3: Linguistic direct relation matrices were arranged in direct relation matrices to express a single matrix of different linguistic expert assessment. Afterwards by taking the arithmetic mean of 88 matrices they were reduced to a single evaluation matrix.

Step 4: The direct relation matrix was normalized as Eq. (2), where Eq. (3) and Eq. (4). Obtained single evaluation matrix was arranged to normalized direct relation matrix using Eqs. (3) and (4).

$$
\tilde{X}=\left[\begin{array}{cccc}
\tilde{x}_{11} & \tilde{x}_{12} & \ldots & \tilde{x}_{1 n} \\
\tilde{x}_{21} & \tilde{x}_{22} & \ldots & \tilde{x}_{2 n} \\
\vdots & \vdots & \ddots & \vdots \\
\tilde{x}_{n 1} & \tilde{x}_{n 2} & \ldots & \tilde{x}_{n n}
\end{array}\right]
$$

where

$$
\tilde{x}_{i j}=\left(\frac{z_{i j, l}}{r_{l}}, \frac{z_{i j, m}}{r_{m}}, \frac{z_{i j, u}}{r_{u}}\right),
$$

and

$$
r_{s}=\max \left(\sum_{i=1,1 \leq i \leq n}^{n} z_{i j, s}\right) \quad \forall s=l, m, u .
$$

Step 5: Total relation fuzzy matrix as seen in Eq. (5) was calculated. At first $\tilde{X}$ matrix is separated to submatrices as $X_{l}, X_{m}, X_{u}$. Let $t_{i j}=\left(t_{i j, l}, t_{i j, m}, t_{i j, u}\right)$ where $t_{i j, s}=X_{s}\left(I-X_{s}\right)^{-1} \forall s=l, m, u$.

$$
\tilde{T}=\left[\begin{array}{cccc}
\tilde{t}_{11} & \tilde{t}_{12} & \ldots & \tilde{t}_{1 n} \\
\tilde{t}_{21} & \tilde{t}_{22} & \ldots & \tilde{t}_{2 n} \\
\vdots & \vdots & \ddots & \vdots \\
\tilde{t}_{n 1} & \tilde{t}_{n 2} & \ldots & \tilde{t}_{n n}
\end{array}\right] .
$$

Step 6: $D_{i}$ and $R_{i}$ vectors as Eq. (6) and Eq. (7) was calculated. ( $D_{i}$ is total row values and $R_{i}$ is total column values of the $\tilde{T}$ matrix.

$$
\begin{aligned}
& \tilde{D}_{i}=\sum_{j=1}^{n} \tilde{t}_{i j} \quad(i=1,2, \ldots, n), \\
& \tilde{R}_{i}=\sum_{i=1}^{n} \tilde{t}_{i j}(j=1,2, \ldots, n) .
\end{aligned}
$$

Step 7: $D_{i}$ and $R_{i}$ vectors were defuzzified using Eq. (8).

$$
\tilde{D}_{i}^{\text {def }} \text { or } \tilde{R}_{i}^{\text {def }}=\left\{\begin{array}{cc}
u-\sqrt{\frac{(u-l)(u-m)}{2}}, & u-m>m-l, \\
\sqrt{\frac{(u-l)(u-m)}{2}}-1, & u-m<m-l, \\
m, & \text { otherwise. }
\end{array}\right.
$$

Step 8: $\left(\tilde{D}_{i}^{\text {def }}-\tilde{R}_{i}^{\text {def }}\right)$ and $\left(\tilde{D}_{i}^{\text {def }}+\tilde{R}_{i}^{\text {def }}\right)$ were calculated. $\left(\tilde{D}_{i}^{\text {def }}+\tilde{R}_{i}^{\text {def }}\right)$ shows how important the criterion is (could be called prominence), $\left(\tilde{D}_{i}^{\text {def }}-\tilde{R}_{i}^{\text {def }}\right)$ shows which criterion is cause and which one is effect (could be called relation) [2]. Also, when the value of $\left(\tilde{D}_{i}^{\text {def }}-\tilde{R}_{i}^{\text {def }}\right)$ is negative the criterion belongs to the effect group, when is positive the criterion belongs to the cause group.

Step 9: The weights of the criteria were calculated using Eq. (9).

$$
w_{i}=\left\{\sqrt{\left(\tilde{D}_{i}^{\text {def }}+\tilde{R}_{i}^{\text {def }}\right)^{2}+\left(\tilde{D}_{i}^{\text {def }}+\tilde{R}_{i}^{\text {def }}\right)^{2}}\right\} .
$$

Step 10: The weights of the criteria were normalized using Eq. (10) [3]:

$$
W_{i}=\frac{w_{i}}{\max _{1 \leq i \leq n}\left(w_{i}\right)} \forall i=1,2, \ldots, n .
$$

As a results of these steps, normalized weights of material selection criteria were calculated and were shown in Table III. According to Table III, the first three important criteria were determined as healthy, strength and reparability.

\subsection{Selecting the best material for dental filling by fuzzy axiomatic design method}

Axiomatic design (AD) was developed to design a product, a system or a process by Suh [4]. The axiomatic 
$D_{i}, R_{i}, \tilde{D}_{i}^{\text {def }}, \tilde{R}_{i}^{\text {def }}, w_{i}$ and $W_{i}$ values.

\begin{tabular}{c|c|c|c|c|c|c|c|c|c|c}
\hline \hline & \multicolumn{3}{|c|}{$D_{i}$} & \multicolumn{3}{|c|}{$R_{i}$} & & & & \\
\cline { 2 - 9 } & $\mathrm{l}$ & $\mathrm{m}$ & $\mathrm{u}$ & $\mathrm{l}$ & $\mathrm{m}$ & $\mathrm{u}$ & $\tilde{D}_{i}^{\text {def }}$ & $\tilde{R}_{i}^{\text {def }}$ & $w_{i}$ & $W_{i}$ \\
\hline Long life & 1.083 & 1.446 & 2.466 & 1.005 & 1.590 & 2.682 & 1.626 & 1.725 & 3.353 & 0.735 \\
Healthy & 1.465 & 1.845 & 2.810 & 1.943 & 2.367 & 3.371 & 2.004 & 2.525 & 4.559 & 1 \\
Reparability & 1.352 & 1.690 & 2.699 & 1.133 & 1.710 & 2.781 & 1.875 & 1.842 & 3.717 & 0.815 \\
Strength & 0.775 & 1.095 & 2.177 & 1.893 & 2.296 & 3.259 & 1.306 & 2.448 & 3.924 & 0.861 \\
Heat resistance & 1.105 & 1.465 & 2.457 & 1.326 & 1.868 & 2.924 & 1.638 & 2.005 & 3.662 & 0.803 \\
Aesthetic & 0.891 & 1.290 & 2.282 & 1.223 & 1.786 & 2.778 & 1.451 & 1.9 & 3.381 & 0.742 \\
Formability & 0.984 & 1.364 & 2.423 & 0.905 & 1.470 & 2.540 & 1.55 & 1.605 & 3.155 & 0.692 \\
Corrosion resistance & 0.567 & 1.078 & 2.176 & 0.327 & 0.748 & 1.798 & 1.236 & 0.919 & 2.179 & 0.478 \\
Biocompatibility & 0.922 & 1.281 & 2.291 & 1.257 & 1.815 & 2.835 & 1.46 & 1.938 & 3.431 & 0.753 \\
Cost-effective & 0.589 & 1.059 & 2.057 & 1.738 & 2.188 & 3.151 & 1.201 & 2.326 & 3.703 & 0.812 \\
Easy access & 1.825 & 2.253 & 3.219 & 0.206 & 0.255 & 1.235 & 2.399 & 0.525 & 3.472 & 0.762 \\
Easy storage & 0.665 & 1.118 & 2.207 & 1.073 & 1.616 & 2.645 & 1.291 & 1.746 & 3.071 & 0.674 \\
Eco-friendly & 1.325 & 1.855 & 2.725 & 0.217 & 0.265 & 1.246 & 1.945 & 0.535 & 2.853 & 0.626 \\
Versatility & 0.787 & 1.252 & 2.371 & 0.313 & 0.874 & 1.937 & 1.429 & 1.008 & 2.474 & 0.543 \\
Rigid & 0.799 & 1.319 & 2.282 & 0.575 & 0.560 & 1.459 & 1.437 & 0.829 & 2.346 & 0.515
\end{tabular}

TABLE IV

Calculated total and weighted total information contents of the five alternative materials.

\begin{tabular}{|c|c|c|c|c|c|c|c|}
\hline Weights & $\begin{array}{c}\text { Multiplicative } \\
\text { inverse } \\
\text { of weights }\end{array}$ & Criteria \alternatives & $\mathrm{Ag}-\mathrm{Sn}$ & $\mathrm{Ni}-\mathrm{Cr}$ & $\mathrm{Co}-\mathrm{Cr}$ & $\mathrm{Au}-\mathrm{Pt}$ & Plastic- $-\mathrm{SiO}_{2}$ \\
\hline 0.735 & 1.360 & Long life & 4.357 & 4.511 & 3.805 & 4.255 & 4.207 \\
\hline 1.000 & 1.000 & Healthy & 4.255 & 4.781 & 4.781 & 4.781 & 4.357 \\
\hline 0.815 & 1.227 & Reparability & 4.511 & 3.805 & 3.805 & 4.511 & 3.805 \\
\hline 0.861 & 1.162 & Strength & 3.805 & 4.781 & 4.781 & 4.781 & 7.303 \\
\hline 0.803 & 1.245 & Heat resistance & 3.805 & 3.805 & 4.511 & 3.805 & 4.781 \\
\hline 0.742 & 1.348 & Aesthetic & 0.000 & 0.000 & 1.000 & 0.000 & 0.000 \\
\hline 0.692 & 1.445 & Formability & 4.781 & 4.781 & 6.322 & 7.303 & 4.781 \\
\hline 0.478 & 2.093 & Corrosion resistance & 4.357 & 4.207 & 3.805 & 6.322 & 4.511 \\
\hline 0.753 & 1.329 & Biocompatibility & 4.781 & 4.781 & 3.805 & 3.805 & 4.511 \\
\hline 0.812 & 1.231 & Cost-effective & 3.805 & 4.511 & 4.781 & 7.303 & 4.218 \\
\hline 0.762 & 1.313 & Easy access & 4.781 & 7.303 & 4.781 & 7.303 & 3.805 \\
\hline 0.674 & 1.485 & Easy storage & 3.805 & 3.805 & 3.805 & 4.207 & 4.781 \\
\hline 0.626 & 1.598 & Eco-friendly & 3.805 & 4.781 & 4.781 & 7.303 & 4.357 \\
\hline 0.543 & 1.843 & Versatility & 4.511 & 4.357 & 4.218 & 4.781 & 4.781 \\
\hline 0.515 & 1.943 & Rigid & 0.585 & 1.000 & 2.322 & 1.000 & 0.000 \\
\hline \multicolumn{3}{|c|}{ Total information content } & 55.944 & 61.210 & 61.304 & 71.460 & 60.200 \\
\hline \multicolumn{3}{|c|}{ Weighted total information content } & 79.388 & 86.342 & 87.031 & 102.477 & 84.584 \\
\hline
\end{tabular}

design method supports a designer or decision maker to provide a scientific basis for designs. One of the important notions in the axiomatic design is an information axiom. It asserts that a design is the best which has minimum information content. Information content is determined by the interaction between the design and system range. Information content of the common range is calculated using Eq. (11) [4]:

$$
I=\log _{2}\left(\frac{\text { System Range }}{\text { Common Range }}\right) .
$$

In our case, the problem data is not certain and it is fuzzy, therefore fuzzy axiomatic design (FAD) method can be used. FAD method allows defining the problem data as a linguistic type. Defined linguistic data are converted into real numbers using a designed triangular fuzzy expression graph. There is not any rule or approach to 
determine a triangular fuzzy expression graph in the literature. Generally, the graphs are determined according to an expert opinion. We determined a graph with the experts for the digitization of the structure of linguistic variables' membership functions. The determined common triangular fuzzy expression design by four specialist dentists considering $\mathrm{C} 1$ (long life) is shown in Fig. 1.

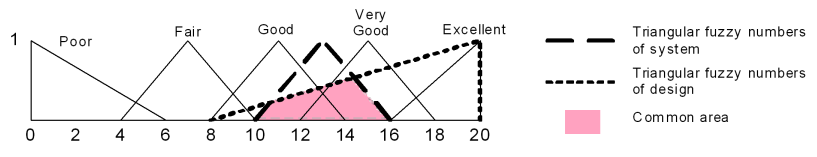

Fig. 1. Common triangular fuzzy expression design considering $\mathrm{C} 1$.

The five alternative filling materials were evaluated considering all criteria separately. Total information contents were calculated according to obtained evaluation data for each alternative material using Eq. (11). Calculated total and weighted total information contents of the five alternative materials are shown in Table IV. According to the results, material $\mathrm{Ag}-\mathrm{Sn}$ should be preferred for dental filling.

\section{Conclusion}

Selecting the best material for dental filling is a difficult process that involves a high level experience. In this study a novel approach was used to select best dental filling material using fuzzy axiomatic design and fuzzy DEMATEL methods. According to dentists' experiences 15 different criteria were determined for 5 different dental filling materials. As a result of the study C2 (healthy) was the most important criterion and $\mathrm{Ag}-\mathrm{Sn}$ is the best material for dental filling. We applied the novel approach on selection dental filling materials. We believe that the proposed approach can be applicable for other material selection problems as well.

\section{References}

[1] E. Fontela, A. Gabus, DEMATEL Report, Battelle Geneva Research Center, Geneva 1976.

[2] J. Jassbi, F. Mohamadnejad, H. Nasrollahzadeh, Expert Syst. Appl. 38, 5967 (2011).

[3] D. Dalalah, M. Hayajneh, F. Batieha, Expert Syst. Appl. 38, 8384 (2011).

[4] N.P. Suh, The Principles of Design, Oxford University Press, New York 1990. 